\title{
Use of thermally induced higher order modes of a microcantilever for mercury vapor detection
}

\author{
Gregory P. Nordin \\ nordin@byu.edu \\ Michael A. George \\ Ashwini R. Kadam
}

Follow this and additional works at: https://scholarsarchive.byu.edu/facpub

Part of the Electrical and Computer Engineering Commons

\section{Original Publication Citation}

Ashwini. R. Kadam, G. P. Nordin, and Michael A. George, "Use of thermally induced higher order modes of a microcantilever for mercury vapor detection," J. Appl Phys. 99, 9495 (26)

\section{BYU ScholarsArchive Citation}

Nordin, Gregory P.; George, Michael A.; and Kadam, Ashwini R., "Use of thermally induced higher order modes of a microcantilever for mercury vapor detection" (2006). Faculty Publications. 976.

https://scholarsarchive.byu.edu/facpub/976

This Peer-Reviewed Article is brought to you for free and open access by BYU ScholarsArchive. It has been accepted for inclusion in Faculty Publications by an authorized administrator of BYU ScholarsArchive. For more information, please contact ellen_amatangelo@byu.edu. 


\title{
Use of thermally induced higher order modes of a microcantilever for mercury vapor detection
}

\author{
Ashwini R. Kadam ${ }^{\text {a) }}$ and Gregory P. Nordin ${ }^{\text {b) }}$ \\ Nano and Micro Devices Center, University of Alabama in Huntsville, Huntsville, Alabama 35899 \\ Michael A. George \\ Department of Chemistry, University of Alabama in Huntsville, Huntsville, Alabama 35899
}

(Received 16 September 2005; accepted 10 March 2006; published online 12 May 2006)

\begin{abstract}
We demonstrate the use of thermally induced higher order modes of a microcantilever as an alternate detection technique by studying $\mathrm{Au}-\mathrm{Hg}$ interactions. The first four thermally induced flexural modes (i.e., the fundamental and first three higher order modes) have been examined for two different Au coatings. In the first case, $50 \mathrm{~nm}$ thick $\mathrm{Au}$ is sputter deposited along the entire length of a rectangular Si microcantilever. In the second case the Au is confined to a $30 \mu \mathrm{m}$ length at the tip of the Si microcantilever. In both cases the microcantilever spectral response and deflection has been studied as a function of $\mathrm{Hg}$ concentration and total exposure time. For the low doses studied, the higher order modes show a distinct $\mathrm{Hg}$ sensing response whereas the fundamental mode does not. The frequency shift for the fourth order mode for a total exposure of $300 \mathrm{~s}$ to approximately $15 \mathrm{ppb} \mathrm{Hg}$ in $\mathrm{N}_{2}$ is found to be -320 and $-70 \mathrm{~Hz}$ for the first and second cases, respectively. (C) 2006 American Institute of Physics. [DOI: 10.1063/1.2194128]
\end{abstract}

\section{INTRODUCTION}

Microcantilevers have been widely studied as chemical and biological sensors in which static bending induced by differential surface stress or changes in resonant frequency upon mass uptake are monitored. ${ }^{1-4}$ In the resonant frequency approach, an externally excited microcantilever is typically used as a highly sensitive mass detector through monitoring a frequency shift. ${ }^{5-7}$ The sensitivity can be further enhanced by increasing the resonant frequency and using a small spring constant. For example, Ono et al. ${ }^{8}$ reduced the microcantilever dimensions and used the resultant higher fundamental frequency to demonstrate mass loading sensitivity in the attogram regime. However, the utility of reducing the microcantilever dimension to increase the fundamental frequency can be limited in practice by the difficulty of obtaining reproducible sensitizing coatings on very small surfaces, as well as the need for a suitable deflection detection technique.

As an alternative, recent efforts have begun to investigate the use of higher order flexural modes of microcantilever vibration. ${ }^{9-13}$ It has been proposed that sensor sensitivity can be improved by using higher order modes for detection because of the associated increase in the frequency of such modes. It has recently been shown ${ }^{11,12}$ that sensor sensitivity using higher order thermal modes should be comparable to reducing the microcantilever length. Moreover, Sharos et al. ${ }^{13}$ indicate that an increase in quality factor is expected for higher order flexural modes compared to the fundamental mode.

In this paper we study the thermally induced fundamental and three higher order modes of Au coated Si microcan-

\footnotetext{
${ }^{a)}$ FAX: (256) 824 2856; electronic mail: kadama@eng.uah.edu

b) Present address: Electrical and Computer Engineering Department, Brigham Young University, Provo, UT 84602.
}

tilevers for $\mathrm{Hg}$ sensing. Previously, $\mathrm{Hg}$ vapor adsorption has been demonstrated on Au coated microcantilevers ${ }^{6,7,14-16}$ using the externally excited fundamental mode of triangular microcantilevers. The effects of $\mathrm{Hg}$ adsorption on the resonant frequency have been reported for both Au coating along the entire microcantilever length and only at the apex. Depending upon the nature of the frequency shifts with $\mathrm{Hg}$ exposure, a qualitative analysis of the effect of mass loading and spring constant changes has been proposed. ${ }^{6,7,13}$ In contrast to these previous studies, we show the possibility of using thermally induced higher order modes of rectangular microcantilevers for $\mathrm{Hg}$ sensing.

Commercial rectangular Si microcantilevers (from Ultrasharp) that are $300 \mu \mathrm{m}$ long and $35 \mu \mathrm{m}$ wide and approximately $1 \mu \mathrm{m}$ thick are used in this study. Two different cases, (i) microcantilevers sputter deposited with $\mathrm{Cr} / \mathrm{Au}$ along the entire length (fully loaded microcantilevers) and (ii) microcantilevers with $\mathrm{Cr} / \mathrm{Au}$ coated only along the last $30 \mu \mathrm{m}$ at the tip (end-loaded microcantilever), have been studied. Upon Hg adsorption, significant shifts in the thermally induced higher order modes have been observed for both cases. The fundamental mode, however, did not show any appreciable frequency shift.

\section{EXPERIMENTAL SETUP}

The experimental setup used for this study is shown in Fig. 1. The laser reflection technique is employed to measure both the microcantilever deflection and resonance spectrum. A Au coated microcantilever is placed in a gas chamber with constant $\mathrm{N}_{2}$ flow and exposed to pulses of $\mathrm{Hg}$ vapor. $\mathrm{A} \mathrm{Hg}$ effusion tube, placed inside a Dynacalibrator (Model 190, Valco Instruments Co. Inc.) at $40{ }^{\circ} \mathrm{C}$, with $32.576 \mathrm{ng} / \mathrm{min}$ effusion rate is used to precisely control the $\mathrm{Hg}$ concentration, which is constantly monitored at the exit of the gas 


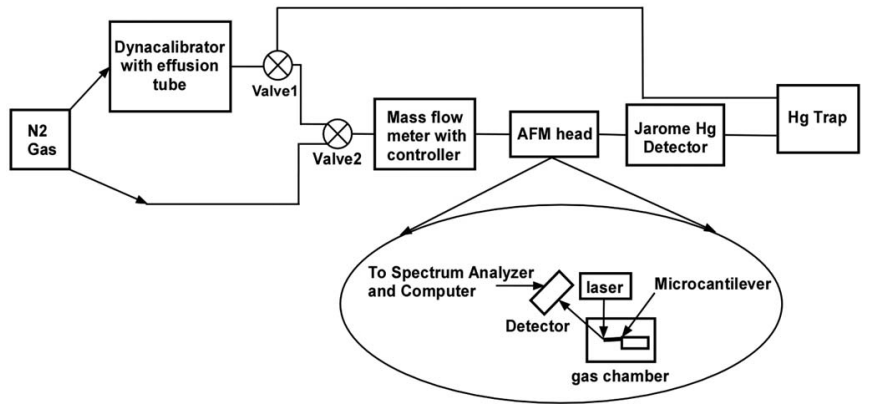

FIG. 1. Experimental setup. Valve 2 is a three-way valve which connects the gas chamber either to nitrogen gas or mercury vapor depending upon its position.

chamber with a Jerome 431-X Hg vapor analyzer (Arizona Instruments, Phoenix, AZ). The deflection of the microcantilever is recorded with a computer controlled data acquisition system and the thermal spectral response is recorded using an HP3588A Spectrum Analyzer.

\section{MICROCANTILEVER THERMAL SPECTRUM}

A typical thermal resonance spectrum of a microcantilever is shown in Fig. 2, where the amplitude of vibration in units of $\mathrm{dBm}$ is plotted on the $y$ axis. The inset shows the analytically calculated equivalent root mean square amplitude in nanometers. Note that four distinct modes are readily discernable. The measured modal frequencies are shown in the last column of Table I. These have been verified to be flexural modes by comparison with the results of three different techniques, as shown in Table I. Analytical calculations based on Refs. 9 and 10 that take into account the effect of the $\mathrm{Au}$ coating ${ }^{17}$ are used to theoretically determine the frequencies and are shown in column 2 of Table I. The third column has resonance frequencies calculated by finite element analysis. In addition, a Digital Instrument Dimension 3000 atomic force microscope (AFM) is used to actively excite the microcantilevers and measure the frequencies. These are shown in the fourth column. Note that the experimental values for the thermally excited modes match very well with the results obtained from the other techniques.

The experimentally calculated quality factors for the four measured modes are shown in the first row of Table II. It can be seen that the quality factor increases approximately

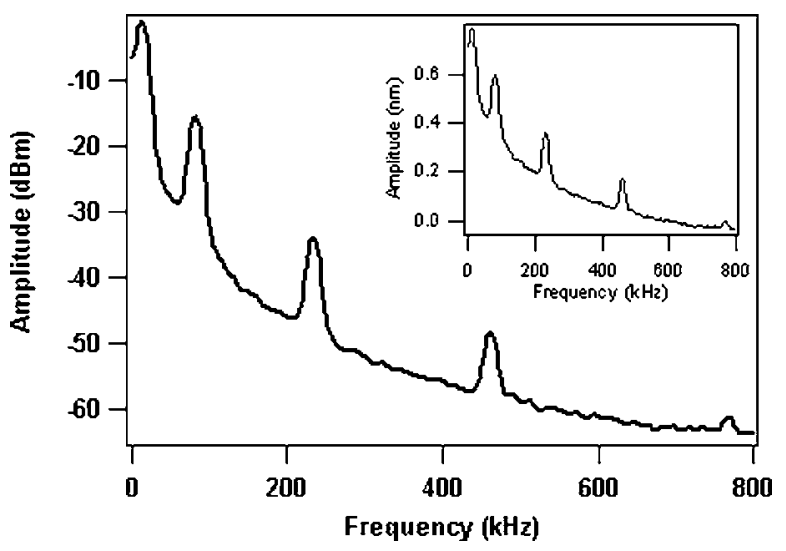

FIG. 2. A typical thermal resonance spectrum of a microcantilever.
TABLE I. Comparison of the values of modal frequencies obtained by different techniques.

\begin{tabular}{ccccc}
\hline \hline Theoretically & & $\begin{array}{c}\text { Frequency } \\
\text { measured with } \\
\text { active } \\
\text { calculated } \\
\text { frequency } \\
\text { Mode }\end{array}$ & $\begin{array}{c}\text { FEM } \\
\text { frequency } \\
(\mathrm{Hz})\end{array}$ & $\begin{array}{c}\text { Experimentally } \\
\text { measured } \\
\text { frequency }(\mathrm{Hz}) \\
\text { D3000 AFM } \\
\text { [observed } \\
\text { standard }\end{array}$ \\
\hline 1 & 12832 & 12786 & 12780 & $12766(0.913)$ \\
2 & 80422 & 80127 & 78870 & $78991(2.754)$ \\
3 & 225185 & 224345 & 225660 & $225990(5)$ \\
4 & 441273 & 439585 & 446160 & $446460(7.071)$ \\
\hline \hline
\end{tabular}

by a factor of 10 when the second order mode is used instead of the first order mode. The quality factor increases by a further factor of 3 when the third order mode is used instead of the second order mode. Similarly in going from the third order mode to the fourth order mode, an approximate increase by a factor of 2 is seen.

As suggested in Refs. 11 and 12, the support loss is significantly different for higher order modes compared to the fundamental mode of microcantilever vibration. Support loss, also known as clamping loss, is the vibration energy of a resonator dissipated by transmission through its support. The quality factor associated with support loss, $Q_{\text {sup }}$, as calculated by Eq. (22) of Ref. 11 is shown in row 3 of Table II for the microcantilevers used in this study. Note that $Q_{\text {sup }}$ increases by approximately a factor of 10, 3, and 2 when going from first to second, second to third, and third to fourth order modes, respectively, which is comparable to what we observe for the measured quality factors. Thus the limitation on the increase in the quality factor of higher order modes appears to be primarily associated with the support loss.

\section{FULLY LOADED MICROCANTILEVER}

To fully load bare Si microcantilevers with $\mathrm{Au}, 5 \mathrm{~nm} \mathrm{Cr}$ and $50 \mathrm{~nm} \mathrm{Au}$ is sputtered on the top surface. The microcan-

TABLE II. Comparison of experimentally observed and theoretical (Refs. 11 and 12) quality factors.

\begin{tabular}{|c|c|c|c|c|}
\hline Mode order $N$ & 1 & 2 & 3 & 4 \\
\hline $\begin{array}{l}\text { Experimental } \\
\text { quality factor }\end{array}$ & 1.475 & 11.177 & 33.078 & 67.770 \\
\hline $\begin{array}{l}\text { Experimental } \\
\text { quality factor }\end{array}$ & $\cdots$ & 7.6 & 3.0 & 2.1 \\
\hline increase $\frac{Q_{N}}{Q_{N-1}}$ & & & & \\
\hline $\begin{array}{l}\text { Calculated } \\
\text { support loss }\end{array}$ & $5.7 \times 10^{6}$ & $4.7 \times 10^{5}$ & $1.7 \times 10^{5}$ & $8.9 \times 10^{4}$ \\
\hline $\begin{array}{l}\text { Theoretically } \\
\text { calculated } \\
\text { increase in } Q_{\text {sup }}\end{array}$ & $\cdots$ & 12.1 & 2.8 & 2.0 \\
\hline$Q_{N}$ & & & & \\
\hline$Q_{N-1}$ & & & & \\
\hline
\end{tabular}

${ }^{\mathrm{a}}$ Reference 11. 


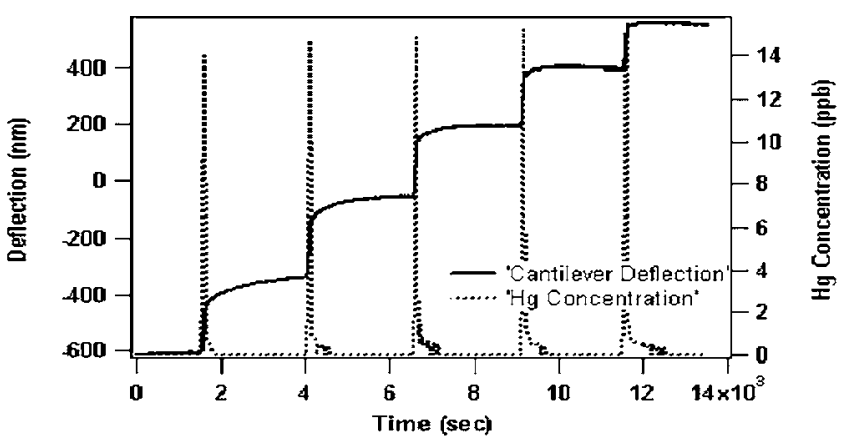

(a)

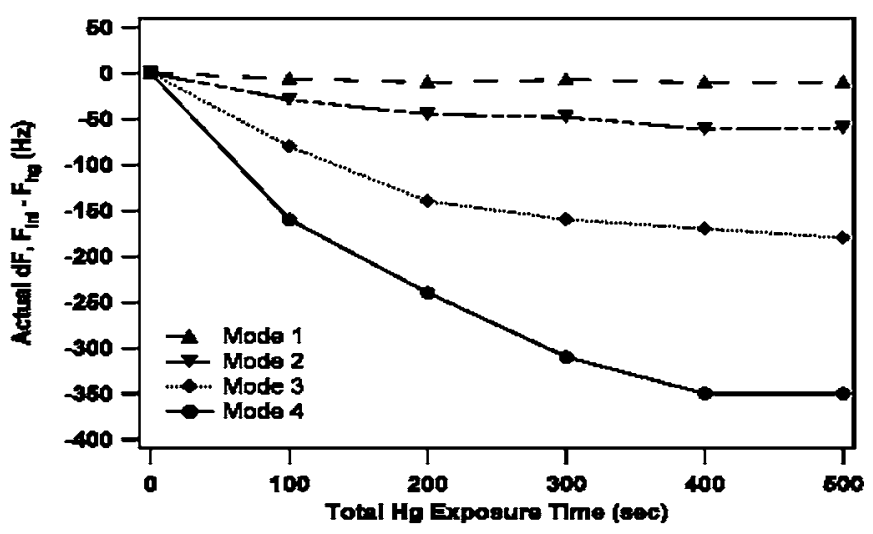

(b)

FIG. 3. (a)Deflection of a fully loaded microcantilever as a function of $\mathrm{Hg}$ exposure time. (b) Spectral response of a fully loaded microcantilever as a function of total $\mathrm{Hg}$ exposure time (difference between the microcantilever's modal frequency before and after $\mathrm{Hg}$ exposure).

tilever is then placed in a gas chamber where the $\mathrm{N}_{2}$ gas flow is maintained at 90 SCCM (SCCM denotes cubic centimeter per minute at STP) and the spectral response is recorded.

After deflection drift stabilization, a microcantilever is exposed to a $15 \mathrm{ppb}$ concentration of $\mathrm{Hg}$ for $100 \mathrm{~s}$. The spectral data is recorded $1000 \mathrm{~s}$ after the $\mathrm{Hg}$ exposure has been stopped. This gives enough time for the deflection signal to stabilize, as shown in Fig. 3(a). Four more subsequent exposures of $\mathrm{Hg}$ are then carried out under identical conditions and the spectral data is taken after each exposure. No saturation in the deflection response of the microcantilever is seen for subsequent $\mathrm{Hg}$ exposures. However, the deflection amplitude decreases slightly with increasing number of exposures. For example, by comparing the microcantilever's deflection amplitude after the first and fifth exposure, approximately $20 \mathrm{~nm}$ decrease in deflection is observed for the last exposure compared with the first. Figure 3(b) shows the frequency shifts (i.e., mode frequency prior to $\mathrm{Hg}$ exposure minus the mode frequency after the $\mathrm{Hg}$ exposure) of the first four flexural modes of the microcantilever as a function of total $\mathrm{Hg}$ exposure time. The fourth order mode undergoes a frequency shift of $-360 \mathrm{~Hz}$ whereas the first order mode shows almost no shift for a total exposure time of $500 \mathrm{~s}$. Note that the frequency shifts of the higher order modes essentially saturate after the fourth $\mathrm{Hg}$ exposure.

The observed frequency shifts for the higher order ther-

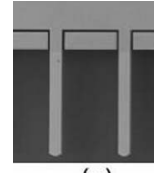

(a)

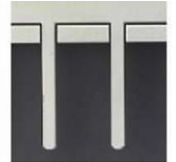

(b)

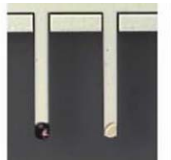

(c)

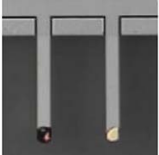

(d)

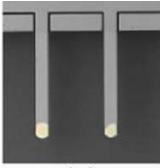

(e)
FIG. 4. Process of end loading the microcantilever: (a) bare Si microcantilever, (b) $5 \mathrm{~nm} \mathrm{Cr}, 50 \mathrm{~nm} \mathrm{Au} \mathrm{coated} \mathrm{microcantilever,} \mathrm{(c)} \mathrm{tip} \mathrm{coating} \mathrm{of}$ photoresist (the contrast between the two photoresist coatings at the tip indicate two different dispensed volumes), (d) after Au etch with photoresist as the etch mask, and (e) end-loaded microcantilevers after photoresist strip and oxygen descum. The $\mathrm{Au}$ area is $1015 \mu \mathrm{m}^{2}$.

mally induced modes are negative for fully loaded rectangular microcantilevers in which the Au layer is sputtered. The fundamental mode shows a shift of only $-3 \mathrm{~Hz}$. In the literature, ${ }^{6,7}$ the externally excited fundamental mode of a fully loaded triangular microcantilever having thermally evaporated $\mathrm{Au}$ as the $\mathrm{Hg}$ sensing surface shows positive frequency shifts, which is opposite to what we report here for higher order modes. Initial experimental results in our laboratory for thermally evaporated versus sputtered $\mathrm{Au}$ films indicate that the difference in sign is related to the interfacial energies associated with the surface morphology of the Au coating, which is different for the two deposition methods. However, this point requires further investigation. At present we are in the process of studying the differences between $\mathrm{Au}$ surfaces for sputtered and thermally evaporated films in the context of $\mathrm{Hg}$ sensing with microcantilevers.

\section{END-LOADED MICROCANTILEVER}

We turn now to an examination of frequency shifts for higher order thermal modes upon $\mathrm{Hg}$ adsorption for endloaded rectangular microcantilevers. To fabricate end-loaded microcantilevers, the use of shadow masks has been proposed. ${ }^{18}$ Here we employ a simple technique, in which the $\mathrm{Au}$ is selectively wet etched with photoresist as an etch mask. Figure 4 shows optical microscope pictures at various stages in the end-loading process. A bare Si microcantilever is first sputter deposited with $5 \mathrm{~nm} \mathrm{Cr}$ and $50 \mathrm{~nm} \mathrm{Au}$. A borosilicate glass micropipette with $10 \mu \mathrm{m}$ inner diameter is used to dispense a known amount of photoresist onto the tip of the microcantilever. The amount dispensed via micropipette is controlled by a nanoliter injector (Nanoliter-2000, World Precision Instruments). The photoresist is then soft baked at $90{ }^{\circ} \mathrm{C}$ for $2 \mathrm{~min}$. The Au is wet etched for $2 \mathrm{~min}$ in $\mathrm{Au}$ etchant (GE-8110, Transene Company Inc.). The photoresist etch mask is stripped in acetone for $5 \mathrm{~min}$. The microcantilever is then subjected to oxygen plasma $(100 \mathrm{~W}$, $100 \mathrm{mT}, 40 \mathrm{SCCM} \mathrm{O}_{2}$ in a PlasmaTherm System 790) for 5 min to remove any residual photoresist.

After measuring the thermal resonance spectrum prior to $\mathrm{Hg}$ exposure, an end-loaded microcantilever is exposed to a $14.5 \mathrm{ppb}$ concentration of $\mathrm{Hg}$ for $100 \mathrm{~s}$. The deflection is continuously monitored [Fig. 5(a)] and the spectral data is recorded $1000 \mathrm{~s}$ after the $\mathrm{Hg}$ exposure. Two more identical $100 \mathrm{~s}$ exposures are carried out. In Fig. 5(b) the frequency shifts of the first four flexural modes of the microcantilever are shown as a function of the total $\mathrm{Hg}$ exposure time. As seen in Fig. 5(b), after a continuous decrease in the fourth 


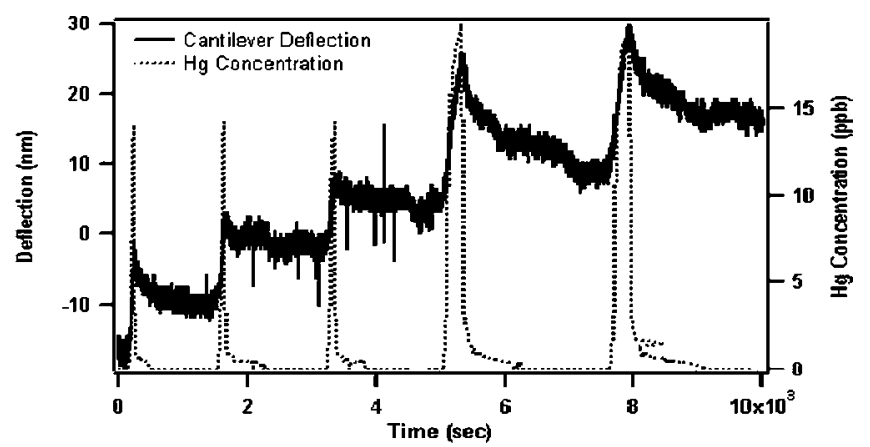

(a)

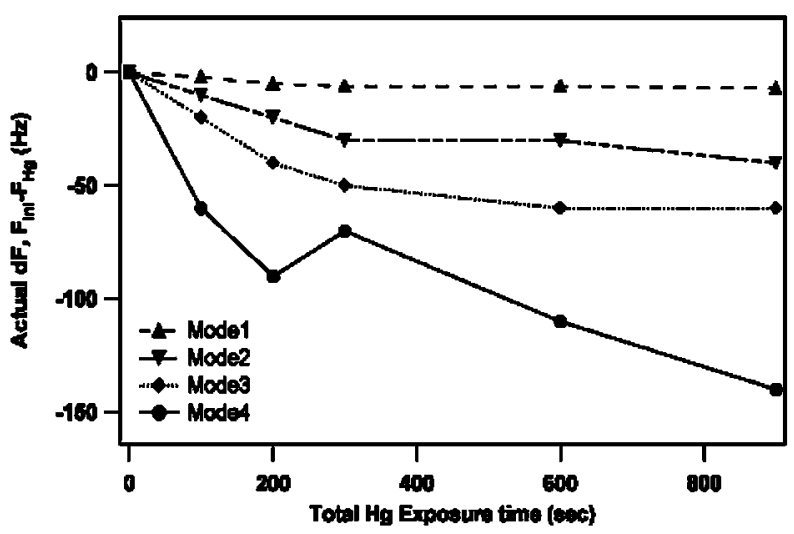

(b)

FIG. 5. (a) Deflection of an end-loaded microcantilever as a function of $\mathrm{Hg}$ exposure time. (b) Spectral response of a fully loaded microcantilever as a function of total $\mathrm{Hg}$ exposure time (difference between the microcantilever's modal frequency before and after $\mathrm{Hg}$ exposure).

order modal frequency, a sudden increase by $20 \mathrm{~Hz}$ after the third $\mathrm{Hg}$ exposure is observed. To check out this discrepancy and to find the nature of the frequency response, two higher concentration exposures $(20 \mathrm{ppb})$, i.e., the fourth and fifth exposures in Fig. 4(a), for a longer time (300 s) were performed. Note that in contrast to the deflection response of the fully loaded microcantilevers, the deflection signal in Fig. 5(a) decreases after the fourth and fifth exposures. This may indicate that some $\mathrm{Hg}$ desorbs from the microcantilever. Also note that the spectral shifts of the second and third order modes almost saturate after the fourth exposure. For a total exposure time of $900 \mathrm{~s}$, the fourth order mode undergoes a frequency shift of $-140 \mathrm{~Hz}$ whereas the first order mode shows almost no frequency shift, similar to the fully loaded microcantilever case.

\section{CONCLUSIONS}

The thermally induced fundamental and first three higher order modes of rectangular microcantilevers have been stud- ied for $\mathrm{Hg}$ sensing. These modes are verified to be the flexural modes of microcantilever vibration. The quality factor associated with these four modes has been analytically calculated and compared with the theory proposed by Hao et $a l .{ }^{12}$ The increase in the quality factor is found to be limited by the support loss of the beam when using higher order modes. Fully loaded and end-loaded microcantilevers exposed to precise concentrations of $\mathrm{Hg}$ show significant shifts in the higher order modal frequencies while the fundamental mode does not show an appreciable shift under the same experimental conditions. For both fully loaded and endloaded microcantilevers with repeated $\mathrm{Hg}$ exposures, the deflection signal does not saturate for the conditions studied. However, the shifts observed in the higher order modes seem to saturate with further exposures. Also, fully loaded microcantilevers show larger frequency shifts for the higher order modes. To fully understand the potential of thermally induced higher order modes as an alternate detection technique, more studies over a large $\mathrm{Hg}$ concentration range and $\mathrm{Hg}$ exposure times are needed.

\section{ACKNOWLEDGMENTS}

The authors would like to thank A. K. Kar and S. Kim for useful discussions. This work was supported in part by DARPA Grant No. N66001-01-1-8933, NSF Grant Nos. EPS-0091853 and ECS-0602261, and NASA EPSCoR Grant No. NCC5-580.

${ }^{1}$ H. Jensenius, J. Thaysen, A. A. Rasmussen, L. H. Veje, O. Hansen, and A. Boisen, Appl. Phys. Lett. 76, 2615 (2000).

${ }^{2}$ T. Thundat and L. Maya, Surf. Sci. 430, L549 (1999).

${ }^{3}$ M. Su, S. Li, and V. P. Dravid, Appl. Phys. Lett. 82, 3562 (2003).

${ }^{4}$ B. Illic, D. Czaplewski, H. G. Craighead, P. Neuzil, C. Campagnolo, and C. Batt, Appl. Phys. Lett. 77, 450 (2000).

${ }^{5}$ H. P. Lang et al., Anal. Chim. Acta 393, 59 (1999).

${ }^{6}$ T. Thundat, E. A. Wachter, S. L. Sharp, and R. J. Warmack, Appl. Phys. Lett. 66, 1695 (1995).

${ }^{7}$ T. Thundat, R. J. Warmack, G. Y. Chen, and D. P. Allison, Appl. Phys. Lett. 64, 2894 (1994).

${ }^{8}$ T. Ono, X. Li, H. Miyashita, and M. Esashi, Rev. Sci. Instrum. 74, 1240 (2002).

${ }^{9}$ T. E. Schaffer and H. Fuchs, J. Appl. Phys. 97, 083524 (2005).

${ }^{10}$ R. Sandberg, W. Svendsen, K. Molhave, and A. Boisen, J. Micromech. Microeng. 15, 1454 (2005).

${ }^{11}$ F. Lochon, I. Dufour, and D. Rebiere, Sens. Actuators B 108, 979 (2005).

${ }^{12}$ Z. Hao, A. Erbil, and F. Ayazi, Sens. Actuators, A 109, 156 (2003).

${ }^{13}$ L. B. Sharos, A. Raman, S. Crittenden, and R. Reifenberger,Appl. Phys. Lett. 84, 4638 (2004).

${ }^{14}$ E. A. Wachter and T. Thundat, Rev. Sci. Instrum. 66, 3662 (1995).

${ }^{15}$ Z. Hu, T. Thundat, and R. Warmach, J. Appl. Phys. 90, 427 (2001).

${ }^{16}$ B. Rogers et al., Rev. Sci. Instrum. 74, 4899 (2003).

${ }^{17}$ J. E. Sader, I. Larson, P. Mulvaney, and L. R. White, Rev. Sci. Instrum. 66, 3789 (1995).

${ }^{18}$ P. I. Oden, Sens. Actuators B 53, 191 (1998). 